\title{
PAPER
}

\section{Continuous monitoring of cerebrovascular autoregulation: a validation study}

\section{E W Lang, H M Mehdorn, N W C Dorsch, M Czosnyka}

See end of article for authors' affiliations

......................

Correspondence to: Dr Erhard W Lang, Department of

Neurosurgery, Level 5 ,

Westmead Hospital,

Sydney, NSW 2145

Australia;

keeflang@optusnet.com.au

Received 13 August 2001

In revised form

21 December 2001

Accepted

10 January 2002
Background: Continuous monitoring of dynamic cerebral autoregulation, using a moving correlation index of cerebral perfusion pressure and mean middle cerebral artery flow velocity, may be useful in patients with severe traumatic brain injury to guide treatment, and has been shown to be of prognostic value.

Objective: To compare an index of dynamic cerebral autoregulation $(\mathrm{Mx})$ with an index of static cerebral autoregulation (sRoR).

Methods: Mx was validated in a prospective comparative study against sRoR, using 83 testing sessions in 17 patients with traumatic brain injury. sRoR and $M x$ were calculated simultaneously during pharmacologically induced blood pressure variations.

Results: Mx was significantly correlated with $\operatorname{RoR}(R=-0.78, \mathrm{p}<0.05)$. Nine patients were found to have failure of cerebral autoregulation, with an sRoR value $<50 \%$. If an $M x$ value of 0.3 was used as the cut off point for failure of cerebral autoregulation, this index had 100\% sensitivity and $90 \%$ specificity for demonstrating failure of autoregulation compared with the sRoR. An increase in cerebral blood flow velocity correlated significantly with $M x(R=0.73, p<0.05)$ but not with cerebral perfusion pressure $(R=0.41)$.

Conclusions: Dynamic and static cerebral autoregulation are significantly correlated in traumatic brain injury. Cerebral autoregulation can be monitored continuously, graded, and reliably assessed using a moving correlation analysis of cerebral perfusion pressure and cerebral blood flow velocity $(M x)$. The $M x$ index can be used to monitor cerebral blood flow regulation. It is useful in traumatic brain injury because it does not require any external stimulus.
C erebral pressure autoregulation is an intrinsic ability of the brain to maintain a stable blood flow in the face of changes in arterial blood pressure or cerebral perfusion pressure. It is a major self defence mechanism against secondary ischaemic insults after traumatic brain injury and subarachnoid haemorrhage. Impairment of cerebral autoregulation has been shown to affect prognosis. ${ }^{1}$ Cerebral autoregulation involves both a response to slow changes in arterial blood pressure or cerebral perfusion pressure, known as "static autoregulation," ${ }^{2}$ and a response to rapid changes in arterial blood pressure or cerebral perfusion pressure, called "dynamic autoregulation." ${ }^{3}$ For patients with severe traumatic brain injury it would be desirable to be able to monitor cerebral autoregulation without recourse to mechanical manipulation of the blood pressure-for example, by the carotid compression and release manoeuvre, ${ }^{4}$ sudden head tilt manoeuvres, ${ }^{5}$ or leg cuff deflation tests ${ }^{6}$ - because these may cause undesirable increases in intracranial pressure, while pharmacological blood pressure manipulations are difficult and time consuming to perform.

With this in mind, one of our group (MC) has developed a cerebral autoregulation monitoring algorithm, known as "Mx", which is based on the continuous analysis of slow, spontaneous fluctuations of cerebral perfusion pressure and cerebral blood flow velocity measured by transcranial Doppler ultrasound. ${ }^{3}$ Because there have been only a few studies assessing the inter-test agreement of cerebral autoregulation tests, ${ }^{7-12}$ and because $\mathrm{Mx}$ has so far only been compared with the leg cuff deflation test, which is also an index of dynamic cerebral autoregulation, we have now compared $\mathrm{Mx}$ with a well established index of static cerebral autoregulation, the static rate of regulation (sRoR). ${ }^{2}$

Both Mx and sRoR can serve as indices to express the stability of cerebral blood flow during changes in cerebral perfusion pressure. The main difference is that the Mx index is usually derived from comparatively small and spontaneous fluctuations of cerebral perfusion pressure and cerebral blood flow velocity, while the sRoR index requires somewhat larger and pharmacologically induced blood pressure variations. In this protocol, calculating $\mathrm{Mx}$ from pharmacologically induced blood pressure variations had a twofold purpose: first, to use an identical dataset for comparison of both indices, and second, to validate the use of Mx as an index of the stability of cerebral blood flow velocity during large variations in cerebral perfusion pressure.

\section{METHODS}

\section{Patients}

Our patient group comprised two women and 15 men. Their mean (SD) age was 43 (15) years. All the patients had a Glasgow coma scale of less than 9 after initial resuscitation on admission, or it deteriorated to that level within the first 12 hours. Further details are shown in table 1.

Blood pressure recordings were obtained using a radial artery fluid coupled system (pvb, Kirchseeon, Germany). Intracranial pressure was measured with an intraparenchymal sensor (Intracranial Pressure Express ${ }^{\circledR}$, Codman, Bracknell, UK; or Camino V420®, San Diego, California, USA). Intracranial pressure sensors were placed on the side of the injury, or in the right frontal area when there was diffuse injury or multiple contusions.

Cerebral blood flow velocities were recorded bilaterally using transcranial Doppler ultrasound (Multi-Dop X2 ${ }^{\circledR}$, DWL, Sipplingen, Germany), and the calculated mean blood flow velocity from both sides for all testing sessions was used. Obtaining a comparable transcranial Doppler signal source on different monitoring occasions was achieved by using a small 


\begin{tabular}{|c|c|c|c|c|}
\hline Patient & $\begin{array}{l}\text { Age } \\
\text { (years) }\end{array}$ & Sex & Injury & Monitoring day* \\
\hline 1 & 46 & M & EDH; bilateral contusions & $4,5,6,7,9,10,11,12,13$ \\
\hline 2 & 37 & M & EDH; SDH; bilateral contusions & $5,6,7,8,12,13$ \\
\hline 3 & 41 & M & SDH & $2,3,4,7$ \\
\hline 4 & 28 & M & Bilateral SDH; EDH & 5,8 \\
\hline 5 & 73 & M & SDH; unilateral contusion & 2,3 \\
\hline 6 & 26 & M & Multiple contusions & $2,3,5,6,8,10$ \\
\hline 7 & 38 & M & EDH & $3,5,6,9$ \\
\hline 8 & 61 & M & Multiple contusions; SDH & $3,4,7$ \\
\hline 9 & 56 & $\mathrm{~F}$ & SDH; multiple contusions & $2,3,5,6,7,9,10$ \\
\hline 10 & 45 & M & Unilateral contusion & $0,1,3,4$ \\
\hline 11 & 26 & M & EDH & $2,3,4,7,8,9,10,15$ \\
\hline 12 & 22 & M & $\mathrm{EDH} ; \mathrm{SDH}$; unilateral contusion & $1,2,4,5,6,8,10$ \\
\hline 13 & 53 & M & SDH; unilateral contusion & $2,4,6$ \\
\hline 14 & 22 & M & EDH & $1,2,3,4,6$ \\
\hline 15 & 59 & M & Bilateral contusions & $1,3,5,7$ \\
\hline 16 & 46 & $\mathrm{~F}$ & Multiple contusions & $2,3,5,8,9,11$ \\
\hline 17 & 53 & M & SDH, multiple contusions & $3,6,7$ \\
\hline
\end{tabular}

rigid head frame (the "Arthur Lam transcranial Doppler probe holder"), which attaches at the nasion and the ears and on which transcranial Doppler probes can be firmly mounted and maintained in a fixed position. Having ensured positional stability in this way, we used the same settings for depth, power, sample volume, and gain at each test session.

The management of these patients consisted of aggressive surgical and medical treatment including immediate evacuation of intracranial mass lesions, mechanical ventilation, and control of intracranial pressure, using a protocol consistent with the Guidelines for the Management of Severe Head Injury. ${ }^{13}$

\section{METHODS}

Our protocol involved manipulating arterial blood pressure with noradrenaline (norepinephrine; Arterenol) to achieve cerebral perfusion pressure changes between approximately 50 and $100 \mathrm{~mm} \mathrm{Hg}$. Eighty three cerebral autoregulation test sessions involving blood pressure variations were undertaken in 17 patients with traumatic brain injury. The minimum cerebral perfusion pressure at each test session was that obtained by decreasing the routine infusion of noradrenaline gradually to zero, or to a point where the perfusion pressure fell to 55 $\mathrm{mm} \mathrm{Hg}$; the noradrenaline infusion was then gradually increased until the perfusion pressure reached approximately $100 \mathrm{~mm} \mathrm{Hg}$, after which it was cut back to maintain a desirable level. The protocol required about 35 to 50 minutes to complete.

Throughout these manipulations, all physiological variables were closely observed; ventilator settings and the levels of sedation (midazolam) and analgesia (fentanyl) were maintained constant during the study. Arterial $\mathrm{PCO}_{2}$ was maintained between 4.7 and $5.1 \mathrm{kPa}$. No additional treatment aimed at controlling intracranial pressure - such as mannitol or barbiturates-was given from 45 minutes before the study until after its completion. In all cases blood pressure manipulations were achieved according to the protocol. During one blood pressure elevation we observed cardiac arrhythmias, but these resolved spontaneously after decreasing the noradrenaline infusion rate.

All analogue signals were recorded, averaged, and stored digitally using a Neurox ${ }^{\circledR}$ multimodality data acquisition system (GMS, Kiel-Mielkendorf, Germany). Fifteen second time-averaged values of arterial blood pressure, mean cerebral blood flow velocity, and mean intracranial pressure were captured and stored on disk for analysis. The cerebral perfusion pressure was calculated on-line as the difference between arterial blood pressure and intracranial pressure. The same data sample was used for the calculation of both Mx and sRoR. The number of testing sessions varied depending on the time of presentation, the evolution of the injury, and the patient's clinical course in the intensive care unit. In order to avoid potential bias caused by different numbers of test sessions, all sessions for each patient were averaged.

This study was performed with the approval of the local university ethics committee, which waived the need for informed consent because varying the cerebral perfusion pressure was considered to be an individual therapeutic trial.

\section{Description of indices}

Mx

This autoregulatory index is a moving correlation coefficient between cerebral perfusion pressure and cerebral blood flow velocity over five minute intervals, and averaged for each investigation and each patient. It represents a mathematical approach to quantifying the relation between spontaneous fluctuations of cerebral perfusion pressure and cerebral blood flow velocity. Based on previous studies, negative values or values less than 0.3 indicate intact cerebral autoregulation, whereby an increase in cerebral perfusion pressure should have no or little effect on cerebral blood flow velocity, while positive values above 0.3 indicate failure of cerebral autoregulation. ${ }^{3}$

\section{Static rate of regulation (sRoR)}

This index describes the change in cerebrovascular resistance (CVR) determined from the relation between cerebral blood flow velocity (CBFV) and changing cerebral perfusion pressure (CPP). It is calculated as

$$
\begin{aligned}
& \text { sRoR }(\%)=100(\% \Delta \mathrm{CVR} / \% \Delta \mathrm{CPP}) \\
& \text { where CVR }=\mathrm{CPP} / \mathrm{CBFV} .{ }^{2}
\end{aligned}
$$

An sRoR of $100 \%$ or more indicates completely intact autoregulation where cerebral blood flow velocity is independent of cerebral perfusion pressure, similar to the plateau phase of cerebral autoregulation; $0 \%$ indicates complete loss of cerebral autoregulation, with cerebral blood flow purely dependent on and linearly related to cerebral perfusion pressure. A value of $50 \%$ is considered the cut off for failure of autoregulation. Thus sRoR is an index expressing quantitatively the stability of changes in cerebral blood flow when arterial blood pressure (or cerebral perfusion pressure) varies. 
Table 2 Mean values and standard deviations of cerebral perfusion pressure (CPP) and cerebral blood flow velocity (CBFV)

\begin{tabular}{lll}
\hline & Baseline & Maximum \\
\hline $\mathrm{CPP}(\mathrm{mm} \mathrm{Hg})$ & $60(10)$ & $95(12)$ \\
$\mathrm{CBFV}(\mathrm{cm} / \mathrm{s})$ & $61(20)$ & $79(17)$ \\
\hline
\end{tabular}

Values are mean $(S D)$

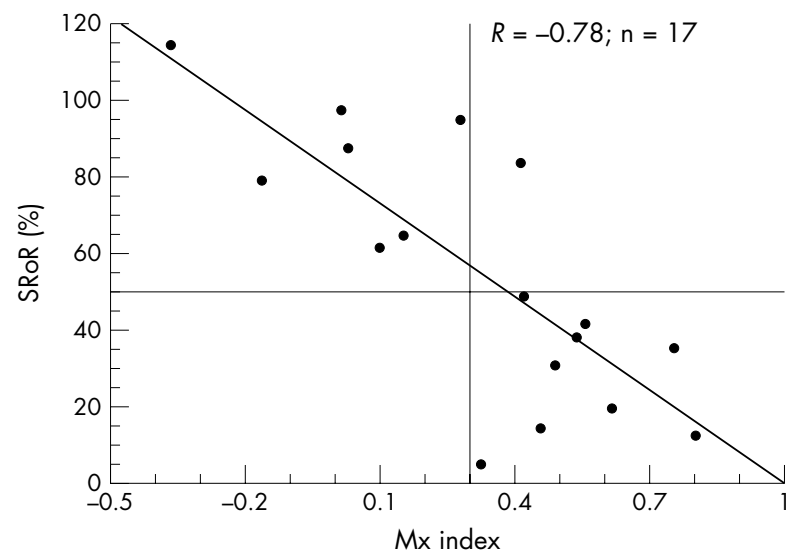

Figure 1 Significant correlation of the $M x$ index with the sRoR index $(R=-0.78, p<0.05)$. Nine patients were found to have failure of cerebral autoregulation, with sRoR values of $<50 \%$, indicated by the points below the horizontal line. When a value of 0.3 was selected as the cut off point for failure of cerebral autoregulation using the $M x$ index-indicated by the points to the right of the vertical line-this index had 100\% sensitivity and $90 \%$ specificity for showing failure of cerebral autoregulation compared with the sRoR index.

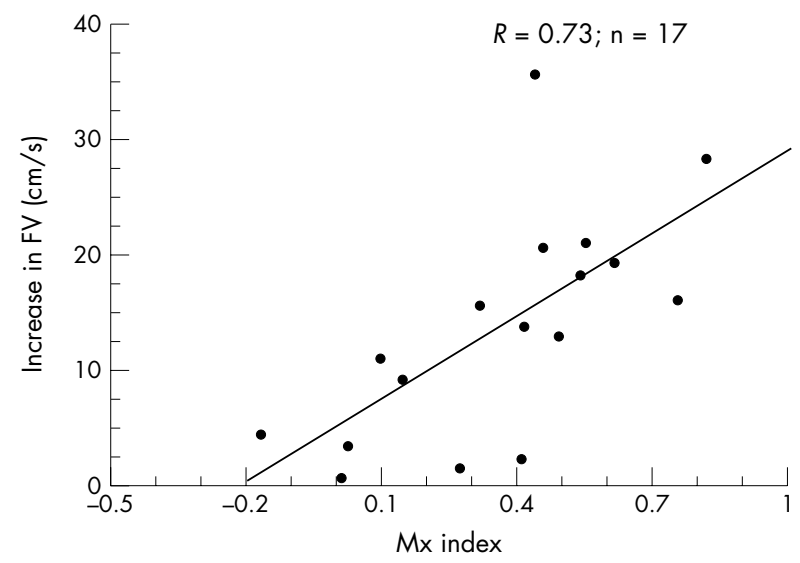

Figure 2 Increased cerebral blood flow velocity (FV) during a rise in blood pressure correlating significantly with the $M x$ index $(R=0.73, \mathrm{p}<0.05)$.

\section{RESULTS}

Table 1 gives information on the patients. During blood pressure variations, cerebral perfusion pressure (mean (SD)) increased from 60 ( 10 ) to 95 (12) $\mathrm{mm} \mathrm{Hg}$. Mean cerebral blood flow velocity increased from 61 (20) to 79 (17) cm/s (table 2).

The Mx index showed a significant correlation with sRoR $(R=-0.78 ; \mathrm{p}<0.05 ;$ fig 1$)$. Nine patients were found to have failure of cerebral autoregulation, with sRoR values of $<50 \%$ (fig 1, below the horizontal line). If 0.3 was used as the cut off point for failure of cerebral autoregulation using the $\mathrm{Mx}$ index (fig 1, right side of the vertical line), this index had 100\% sensitivity and, with one false positive, $90 \%$ specificity for demonstrating failure of cerebral autoregulation compared with the sRoR.

At the same time an increase in the cerebral blood flow velocity correlated significantly with the $\mathrm{Mx}(R=0.73$, $\mathrm{p}<0.05$; fig 2) but not with cerebral perfusion pressure $(R=0.41 ; \mathrm{p}=0.08$, NS). This shows that the greater the increase in blood flow velocity during cerebral perfusion pressure changes, the greater the value of $\mathrm{Mx}$.

\section{DISCUSSION}

Our study shows that cerebral autoregulation can be monitored continuously, graded, and reliably assessed using a correlation analysis of slow cerebral blood flow velocity waves and cerebral perfusion pressure, known as Mx. Although measured for the purposes of this study at the same time as sRoR, the Mx index does not normally require any external mechanical or pharmacological stimuli. Mx serves to indicate and quantify the stability of cerebral blood flow regulation during blood pressure changes. This interpretation is based on a physiological model in which intact cerebral autoregulation is indicated by an autoregulatory plateau phase, whereby changing the cerebral perfusion pressure has little effect on cerebral blood flow velocity. ${ }^{14}$ Our study also shows that dynamic and static assessments of cerebral autoregulation are significantly correlated in patients with traumatic brain injury.

\section{Continuous cerebral autoregulation monitoring}

While previous comparative studies required repeated testing sessions, this study used identical datasets for simultaneous assessment of static and dynamic cerebral autoregulation. Subsequent or repeated cerebral autoregulation testing may have influenced the results to an unknown extent in previous studies. So far only the leg cuff deflation test has been shown to yield stable results on repetition. ${ }^{15}$

The Mx monitoring protocol allows continuous monitoring of cerebral autoregulation, while other tests offer only intermittent "snapshot" monitoring. It was pointed out by Lewis et al that autoregulatory disturbance precedes autoregulatory failure. ${ }^{12}$ Continuous $\mathrm{Mx}$ monitoring in patients with traumatic brain injury could thus identify disturbances of cerebral autoregulation in time to achieve successful treatment. The use of continuous cerebral autoregulation monitoring as part of a head injury treatment protocol is supported by Mascia et al, who reported that management of cerebral perfusion pressure with vasopressor agents was safe so long as autoregulation was preserved. They stressed that "the assessment of pressure autoregulation should be considered as a guide for arterial pressure oriented therapy after head injury."16

We have also confirmed that Mx serves as an indicator of the stability of cerebral blood flow regulation during blood pressure changes, being significantly correlated with the sRoR index. The sRoR, however, requires pharmacological induction of blood pressure variations and it is thus more difficult and time consuming to perform. Figure 2 shows the relation between Mx and cerebral blood flow velocity changes: a high Mx signifies a marked change in cerebral blood flow velocity during variation in blood pressure, indicating compromised cerebral autoregulation or complete failure of autoregulation.

\section{Comparative studies}

Inter-test agreement has been examined in only six studies to our knowledge-in traumatic brain injury, ${ }^{9}$ in traumatic brain injury with subarachnoid haemorrhage ${ }^{10}$ in normal subjects during anaesthesia with propofol followed by isoflurane, ${ }^{7}$ in patients with occlusive cerebrovascular disease, ${ }^{8}$ in acute ischaemic stroke, ${ }^{17}$ and in healthy volunteers at different levels of ventilation. ${ }^{11}$ It appears from these studies that there is 
at least some similarity between tests that assess dynamic cerebral autoregulation and those that assess static autoregulation

Smielewski et al reported a significant correlation between the transient hyperaemic response test and the dynamic cerebral autoregulation index, based on a moving correlation analysis between cerebral perfusion pressure and systolic cerebral blood flow velocity in patients with traumatic brain injury (the "Sx" index, contrasted with the mean velocity in our Mx index). ${ }^{9}$ There is also evidence that metabolic cerebral autoregulation correlates well with dynamic cerebral auto regulation assessed by the Valsalva manoeuvre. ${ }^{8}$ Steinmeier et al, however, found no correlation between the orthostatic hypotension test, the cuff deflation test, and the transient hyperaemic response test in a combined subarachnoid haemorrhage/traumatic brain injury group, although there was good agreement between the orthostatic hypotension test and a cross correlation analysis. ${ }^{10}$ In a series of 61 patients with acute stroke, Dawson et al reported that dynamic but not static cerebral autoregulation was impaired. ${ }^{17}$

Piechnik et al compared the Mx and Sx indices at different $\mathrm{CO}_{2}$ levels with the cuff deflation test in healthy volunteers, and reported a "...reasonably good correlation" between both $\mathrm{Mx}$ and Sx and the cuff deflation test. ${ }^{11}$ They stressed that all indices of dynamic cerebral autoregulation depended on the degree of ventilation, hypoventilation causing impairment of autoregulation; this was also shown in the original cuff deflation study. ${ }^{6}$ For intensive care management this finding emphasises the importance of maintaining a constant mild to moderate degree of hyperventilation, which was done in all patients in our study.

Tiecks et al have shown that in normal human subjects measurements of dynamic autoregulation yield similar results to static testing during both intact and pharmacologically impaired autoregulation. ${ }^{7}$ They also suggested that static cerebral autoregulation may be less vulnerable than dynamic autoregulation because of different control mechanisms and centres. Our study shows that in patients with traumatic brain injury dynamic and static cerebral autoregulation are equally affected, which does not allow any conclusions to be drawn about possible control mechanisms.

\section{Conclusions}

Our study provides further insight into the correlation between static and dynamic cerebral autoregulation in severely head injured patients. It confirms the feasibility and value of the $\mathrm{Mx}$ index for continuous and reliable monitoring of cerebral autoregulation. Further comparative studies are needed to determine whether static and dynamic cerebral autoregulation are equally affected in other critical neurosurgical conditions such as subarachnoid haemorrhage or spontaneous hypertensive intracerebral haemorrhage.

\section{ACKNOWLEDGEMENTS}

Our sincere thanks to the nursing staff of the neurosurgical intensive care unit in Kiel for their support and cooperation during this investigation. EWL was supported by grant No La $9162 / 1$ from the Deutsche Forschungsgemeinschaft, Bonn, Germany. MC is on unpaid leave from the Warsaw University of Technology, Poland.

\section{Authors' affiliations}

E Lang, H M Mehdorn, Department of Neurosurgery,

Christian-Albrechts-Universität, Weimarer Strasse 8, D-24106 Kiel, Germany

N W C Dorsch, Department of Neurosurgery, University of Sydney, Westmead Hospital, Level 5, Westmead NSW 2145, Australia M Czosnyka, Academic Neurosurgery Unit and Wolfson Brain Imaging Centre, University of Cambridge, Addenbrooke's Hospital, Box 167, Level 4, A Block, Cambridge CB2 2QQ, UK

\section{REFERENCES}

1 Czosnyka M, Smielewski P, Piechnik S, et al. Cerebral autoregulation following head injury. J Neurosurg 2001;95:756-63.

2 Strebel S, Lam AM, Matta B, et al. Dynamic and static cerebral autoregulation during isoflurane, desflurane, and propofol anesthesia. Anesthesiology 1995;83:66-76.

3 Czosnyka M, Smielewski P, Kirkpatrick P, et al. Monitoring of cerebra autoregulation in head-injured patients. Stroke 1996;27:1829-34.

4 Giller CA. A bedside test for cerebral autoregulation using transcranial Doppler ultrasound. Acta Neurochir (Wien) 1991;108:7-14.

5 Heckmann JG, Hilz M, Hagler H, et al. Transcranial Doppler sonography during acute 80 degrees head-down tilt (HDT) for the assessment of cerebral autoregulation in humans. Neurol Res 1999:21:457-62.

6 Aaslid R, Lindegaard KF, Sorteberg W, et al. Cerebral autoregulation dynamics in humans. Stroke 1989;20:45-52.

7 Tiecks FP, Lam AM, Aaslid R, et al. Comparison of static and dynamic cerebral autoregulation measurements. Stroke 1995;26:1014-19.

8 Tiecks FP, Douville C, Byrd S, et al. Evaluation of impaired cerebral autoregulation by the Valsalva maneuver. Stroke 1996:27:1 177-82.

9 Smielewski P, Czosnyka M, Kirkpatrick P, et al. Evaluation of the transient hyperemic response test in head-injured patients. J Neurosurg 1997;86:773-8.

10 Steinmeier R, Hofmann R, Bauhuf $C$, et al. Continuous autoregulation monitoring in comatose patients [abstract]. J Neurosurg 1999; $90: 427-8$ A

11 Piechnik SK, Yang X, Czosnyka M, et al. The continuous assessment of cerebrovascular reactivity: a validation of the method in healthy volunteers. Anesth Analg 1999:89:944-9.

12 Lewis SB, Wong ML, Bannan PE, et al. Transcranial Doppler identification of changing autoregulatory thresholds after autoregulatory impairment. Neurosurgery 2001;48:369-75 [discussion, pp 375-6].

13 Bullock R, Chesnut RM, Clifton G, et al. Guidelines for the management of severe head injury. New York: Brain Trauma Foundation, 1996.

14 Larsen FS, Olsen KS, Hansen BA, et al. Transcranial Doppler is valid for determination of the lower limit of cerebral blood flow autoregulation. Stroke 1994;25:1985-8.

15 Mahony PJ, Panerai RB, Deverson ST, et al. Assessment of the thigh cuff technique for measurement of dynamic cerebral autoregulation. Stroke 2000;31:476-80.

16 Mascia L, Andrews PJ, McKeating EG, et al. Cerebral blood flow and metabolism in severe brain injury: the role of pressure autoregulation during cerebral perfusion pressure management. Intensive Care Med 2000;26:202-5

17 Dawson SL, Blake M, Panerai RB, et al. Dynamic but not static cerebral autoregulation is impaired in acute ischaemic stroke. Cerebrovasc Dis 2000; 10: 126-32. 